\begin{tabular}{|c|l|}
\hline Title & $\begin{array}{l}\text { Divergence of relative difference in Gaussian distribution function and stochastic resonance in a bistable system with } \\
\text { frictionless state transition }\end{array}$ \\
\hline Author(s) & Kasai, Seiya; Ichiki, A kihisa; Tadokoro, Y ukihiro \\
\hline Citation & $\begin{array}{l}\text { A pplied Physics Express (A PEX), 11/3), 037301 } \\
\text { https://doi.org/10.7567/APEX.11.037301 }\end{array}$ \\
\hline Issue Date & 2018-03 \\
\hline Doc URL & http://hdl.handle.net/2115/72796 \\
\hline Rights & O2018 The Japan Society of A pplied Physics \\
\hline Type & article (author version) \\
\hline File Information & APEX GaussianBistable.pdf \\
\hline
\end{tabular}

Instructions for use 


\section{Divergence of relative difference in Gaussian distribution function and stochastic resonance in a bistable system with frictionless state transition}

Seiya Kasai ${ }^{1, *}$, Akihisa Ichiki ${ }^{2}$, and Yukihiro Tadokoro ${ }^{3}$

${ }^{1}$ Research Center for Integrated Quantum Electronics and Graduate School of Information Science \& Technology, Hokkaido University, North 13, West 8, Sapporo 0608628, Japan

${ }^{2}$ Green Mobility Research Institute, Institutes of Innovation for Future Society, Nagoya University, Furo-cho, Chikusa-ku, Nagoya, 464-8603, Japan

${ }^{3}$ Toyota Central R\&D Laboratories, 41-1, Yokomichi, Nagakute, Aichi 480-1192, Japan

E-mail: kasai@rciqe.hokudai.ac.jp

A bistability system efficiently detects a weak signal by adding noise, which is referred to as stochastic resonance. A previous theory deals with friction in state transition; however, this hypothesis is inadequate when friction force is negligible such as in nanoand molecular-scale systems. We show that, when the transition occurs without friction, the sensitivity of the bistable system to a Gaussian-noise-imposed weak signal becomes significantly high. The sensitivity is determined by the relative difference in noise distribution function. We find that the relative difference in Gaussian distribution function diverges in its tail edge, resulting in a high sensitivity in the present system. 
Stochastic resonance was introduced in 1981 for the mechanism of 100,000-year cycle of the ice age on Earth, where bistability arises from the positive feedback between the surface temperature and solar reflection. ${ }^{1)}$ This planetary-scale mechanism was found to involve in various biological functions. ${ }^{2,3)}$ The unique feature of stochastic resonance has attracted interest in diverse fields and, nowadays, it is known to occur in various micro- and nanoscale systems. ${ }^{4-12)}$ The essence of the phenomenon has also been of interest; a previous theory for a bistable system indicates that the sensitivity to the noiseadded signal is maximized when the signal period matches the time scale of the transition involving the noise intensity, friction, and size of the potential inside the system. ${ }^{11-17)}$ As a result, the output signal-to-noise ratio (SNR) is given as $S N R \propto(s / \sigma)^{2} \exp \left(-U_{0} / \sigma\right)$, where $s$ is the signal amplitude, $\sigma$ is the standard deviation of the noise, and $U_{0}$ is the potential barrier height. ${ }^{18)}$ The exponential part of the formula suggesting a positive contribution of noise is attributed to the Kramers rate, describing the transition with Brownian motion surmounting a barrier. ${ }^{19-20)}$ However, in nano- and molecular-scale systems, the state transition is promptly accomplished without friction, and the Kramerstype description is inadequate. ${ }^{21)}$ This suggests that the behavior of stochastic resonance in such systems distinctively changes from the in the previous system. In this Letter, we show that the bistable system exhibits a significantly high sensitivity to the Gaussian noise-imposed weak signal in terms of stochastic resonance, when the effect of friction is ignorable in state transition. The sensitivity is theoretically derived, which clarifies the effect of the noise characteristics on the response. Then, the unique nonlinear behavior of the Gaussian distribution function results in the high sensitivity at a high input threshold.

Figures 1(a) and 1(b) schematically show the state transitions in the bistable systems without and with friction, respectively. The frictionless transition is often seen in a very small system where the two equivalent states are close to each other. In addition, in such case, each state is strongly confined and it is with less degree of freedom of movement, almost being a two-state system. ${ }^{14,17,22)}$ In our model, we assume that the friction coefficient in transition is zero, and the relaxation immediately occurs after the transition. Then, the state transition promptly occurs whenever the external force lifts up the state to the top of the barrier dividing the states. Figure 1(c) schematically shows the input and output waveforms in this system. The input has two thresholds, $\theta_{\mathrm{L}}=-\theta$ and $\theta_{\mathrm{H}}=\theta(\theta>$ 0 ), and the output value is changed when the signal crosses the input threshold in the 
counter position. We assign the values 0 and 1 to the low and high states in the output, respectively.

When the input is stochastic, the states are randomly switched and the output exhibits a random pulse train. As shown in Fig. 1(c), the transition probabilities, $p_{\mathrm{HL}}$ for high to low transition and $p_{\mathrm{LH}}$ for low to high transition, are evaluated on the basis of the probabilities of the noise-imposed signals crossing $\theta_{\mathrm{L}}$ and $\theta_{\mathrm{H}}$, respectively. When a small input signal $s$ is given to the system and noise $\xi$ is imposed to the signal, $p_{\mathrm{HL}}$ and $p_{\mathrm{LH}}$ are obtained as follows:

$$
\begin{aligned}
& p_{\mathrm{LH}}(s)=\int_{\theta-s}^{\infty} \rho(\xi) d \xi \\
& p_{\mathrm{HL}}(s)=\int_{-\infty}^{-\theta-s} \rho(\xi) d \xi
\end{aligned}
$$

Here, $\rho(\xi)$ is the noise distribution function, which has the same dimension as $1 / s$ and $1 / \xi$. In the case of the white noise with the bandwidth $B W_{\mathrm{N}}$, the average resident times in the high and low states, $\left\langle\tau_{\mathrm{H}}\right\rangle$ and $\left\langle\tau_{\mathrm{L}}\right\rangle$, respectively, are directly connected to $p_{\mathrm{HL}}$ and $p_{\mathrm{LH}}$ such as $\left\langle\tau_{\mathrm{H}}\right\rangle=1 /\left(\alpha \cdot 2 B W_{\mathrm{N}} \cdot p_{\mathrm{HL}}\right)$ and $\left\langle\tau_{\mathrm{L}}\right\rangle=1 /\left(\alpha \cdot 2 B W_{\mathrm{N}} \cdot p_{\mathrm{LH}}\right)$. The coefficient $\alpha$ represents the effect of the noise autocorrelation on the state transition and the output. From the fact that the period of the output pulse $\left\langle\tau_{\mathrm{L}}\right\rangle+\left\langle\tau_{\mathrm{H}}\right\rangle$ is greater than $1 / B W_{\mathrm{N}}$ and the transition probabilities in Eq. (1) are less than $1 / 2, \alpha$ is expected to be 2 . On the other hand, each of $\left\langle\tau_{\mathrm{L}}\right\rangle$ and $\left\langle\tau_{\mathrm{H}}\right\rangle$ is greater than $1 / B W_{\mathrm{N}}$ as the noise crosses the high and low thresholds in an uncorrelated manner; then, $\alpha$ is unity. The expected output value is expressed as $\langle y\rangle$ $=\left\langle\tau_{\mathrm{H}}\right\rangle /\left(\left\langle\tau_{\mathrm{L}}\right\rangle+\left\langle\tau_{\mathrm{H}}\right\rangle\right)$, corresponding to the average duty ratio. Note that $\alpha$ is canceled out by considering the ratio of $\left\langle\tau_{\mathrm{L}}\right\rangle$ to $\left\langle\tau_{\mathrm{H}}\right\rangle$ and that it does not appear in $\langle y\rangle$. When $s$ is sufficiently small, $p_{\mathrm{HL}}$ and $p_{\mathrm{LH}}$ can be characterized by first-order approximation. Then $<y>$ is obtained as follows:

$$
\langle y\rangle \approx \frac{1}{2}\left[1+\frac{\rho(\theta) s}{\int_{\theta}^{\infty} \rho(\xi) d \xi}\right]
$$

In the present system, the output is given by the relative change in transition probability induced by $s$ and is completely dominated by the noise distribution function. This kind of dependence of the output on the noise property has not been seen in a previous model, 
although the output of a single threshold system is dominated by the noise distribution function. ${ }^{23-26)}$

Next, we derive the sensitivity $d\langle y\rangle / d s$ in the case of a Gaussian noise, which deals with almost all random processes in every field. The distribution function of the Gaussian noise is $\rho(\xi)=\left(2 \pi \sigma^{2}\right)^{-1 / 2} \exp \left(-\xi^{2} / 2 \sigma^{2}\right)$. To clarify the input threshold dependence of the sensitivity, the limit of the sensitivity for a high threshold is evaluated. By applying l'Hôpital's rule, the limit is evaluated as follows:

$$
\lim _{\theta \rightarrow \infty} \frac{d\langle y\rangle}{d s}=\lim _{\theta \rightarrow \infty} \frac{\rho(\theta)}{2 \int_{\theta}^{\infty} \rho(\xi) d \xi}=\lim _{\theta \rightarrow \infty} \frac{\rho^{\prime}(\theta)}{2 \rho(\theta)}=\lim _{\theta \rightarrow \infty} \frac{\theta}{2 \sigma^{2}}=\infty
$$

The obtained equation reveals that the sensitivity to the Gaussian-noise-imposed weak signal becomes extremely high when the input threshold is sufficiently high. This behavior is not seen in the previous theory of stochastic resonance in a bistable system. ${ }^{13-}$ 17) However, the high sensitivity at the high input threshold is counterintuitive, because the bell-shaped configuration of the Gaussian function suggests that both $\rho(\theta)$ and $\rho^{\prime}(\theta)$ converge to zero at $\theta \rightarrow \infty$.

An important aspect of the present model is that the origin of the high sensitivity to the noise-imposed weak signal is included in the noise itself. Let us consider the difference between the two adjacent values in the Gaussian function with a small interval $\delta(>0)$. It is easily found that their difference converges to zero in a tail of the function; $\Delta \rho(\theta)=\rho(\theta-\delta)-\rho(\theta) \rightarrow 0$ at $\theta \rightarrow \infty$. On the other hand, when the difference is normalized by $\rho(\theta)$, it diverges to infinity; $\Delta \rho(\theta) / \rho(\theta)=\rho(\theta-\delta) / \rho(\theta)-1=\exp [(2 \theta \delta-$ $\left.\left.\delta^{2}\right) / 2 \sigma^{2}\right]-1 \rightarrow \infty$ at $\theta \rightarrow \infty$. This divergence is unexpected from the configuration of the Gaussian function, because both $\Delta \rho$ and $\rho(\theta)$ converge to 0 in its tail edge. However, the exponent of the Gaussian function including a quadratic term of $\xi$ changes the situation. The distinguished behavior of the Gaussian distribution is shown in Fig. 1(d), where $\Delta \rho / \rho$ is reflected in $\left\langle\tau_{\mathrm{H}}\right\rangle \mid\left\langle\tau_{\mathrm{L}}\right\rangle$ through Eqs. (2) and (3). The Gaussian distribution can achieve a significant increase in $\left\langle\tau_{\mathrm{H}}\right\rangle \mid\left\langle\tau_{\mathrm{L}}\right\rangle$ by a small input signal as the threshold is increased, whereas those of the Cauchy and Logistic distributions are saturated. Therefore, the origin of the high sensitivity in the present system is the nonlinearity of the exponent in the noise distribution function. The generalized condition of the noise achieving the high sensitivity 
to the weak signal is that $\rho(\xi)$ satisfies $\rho^{\prime}(\xi) / \rho(\xi)=d[\ln \rho(\xi)] / d \xi \rightarrow \infty$ at $\xi \rightarrow \infty$. Thus, we can use any noise with a distribution $\rho(\xi)=\exp (-u(\xi)) / Z$ ( $Z$ is the normalization constant) with a function $u(\xi)$ whose derivative $d u / d \xi$ diverges to infinity at $\xi \rightarrow \infty$.

The validity of the present model was experimentally examined. To represent the ideal system in a simple way, we used an electronic two-state device, Schmitt trigger, ${ }^{27)}$ with a sufficiently fast response that could effectively reproduce the frictionless transition. The stochastic resonance in the Schmitt trigger was already demonstrated experimentally $^{28)}$ and several mathematical models for the phenomenon in this device have been developed so far. ${ }^{29,30)}$ However, the analysis used the Kramers rate and did not suggest the high sensitivity indicated in this study. The noise imposed on the signal was white Gaussian noise whose bandwidth $B W_{\mathrm{N}}$ was $1 \mathrm{MHz}$. The switching delay of the device, $\tau_{\mathrm{D}}$, was $30 \mathrm{~ns}$, which is sufficiently smaller than the noise autocorrelation time $\tau_{\mathrm{AC}}=1 / B W_{\mathrm{N}}(\mathrm{s})$. Thereby the device could respond to each impulse of the noise. The signal $s(\mathrm{~V})$ was a DC voltage or a sinusoidal wave with frequency $f_{\mathrm{S}}$ of typically $2 \mathrm{kHz}$, which is sufficiently smaller than the noise bandwidth. The output of the device showed a hysteresis with two input thresholds as shown in Fig. 2(a). The input threshold values $\theta(\mathrm{V})$ and $-\theta(\mathrm{V})$ in the device were changed by varying the supply voltage to the Schmitt trigger, $V_{\mathrm{DD}}(\mathrm{V})$. The input signal was initially biased at the center of the two thresholds.

When the noise was imposed to the input signal, the output showed a random pulse train [Fig. 2(a)]. As shown in Fig. 2(b), the measured resident time had a unique value at each noise intensity. Both $\left\langle\tau_{\mathrm{H}}\right\rangle$ and $\left\langle\tau_{\mathrm{L}}\right\rangle$ quickly decreased as the noise intensity was increased, and asymptotically approached half of the noise-autocorrelation time, $1 / 2 B W_{\mathrm{N}}$. When $s=0 \mathrm{~V},\left\langle\tau_{\mathrm{H}}\right\rangle$ and $\left\langle\tau_{\mathrm{L}}\right\rangle$ were always the same regardless of noise intensity. When $s=0.05 \mathrm{~V},\left\langle\tau_{\mathrm{H}}\right\rangle$ increased while $\left\langle\tau_{\mathrm{L}}\right\rangle$ decreased. The difference was more than two orders at $\sigma=0.1 \mathrm{~V}$, which is quite sensitive to the input signal. The behavior was also changed when the input threshold value was changed and it could be well explained by the theory based on Eq. (1) with $\alpha=1.5$. In this experiment, $\alpha$ in the theoretical resident times took an intermediate value between 1 and 2, because the standard deviation of the noise was close to the threshold value, $\sigma \sim \theta$. We separately confirmed that the noise under this condition crossed both high and low input thresholds frequently, and the width 
and interval of the output pulses were often $1 / 2 B W_{\mathrm{N}}$.

Figure 2(c) shows the ratio of the resident times, $\left\langle\tau_{\mathrm{H}}\right\rangle \mid\left\langle\tau_{\mathrm{L}}\right\rangle$. At $s=0 \mathrm{~V}$, the ratio was always unity regardless of noise intensity, suggesting that the output $\langle y\rangle$ did not depend on the noise intensity at all. This behavior is available as long as the noise distribution is symmetric, and then any change in output is attributed to the input signal $s$. Indeed, $\left\langle\tau_{\mathrm{H}}\right\rangle \mid\left\langle\tau_{\mathrm{L}}\right\rangle$ sensitively changed depending on $s$ as shown in Fig. 2(d). $\left\langle\tau_{\mathrm{H}}\right\rangle \mid\left\langle\tau_{\mathrm{L}}\right\rangle$ under the small signal exponentially increased with increasing input threshold as indicated in Fig. 2(d). This behavior is not seen in the previous theory, ${ }^{16)}$ where the state transition is controlled described by the Kramers rate. ${ }^{13,20)}$ Some deviation between the experimental result and the theory in the high-input-threshold region was attributed to the slight distortion of the noise distribution function observed in the measurement circuit.

The measured transfer characteristics for the Gaussian noise-imposed DC signal are shown in Figs. 3(a) and 3(b). When $s$ was small, the output $\langle y\rangle$ was proportional to $s$, whereas the output saturated at unity when $s$ was large. The saturation meant that the system almost remained at the high level because of $\left.\left.\left\langle\tau_{\mathrm{H}}\right\rangle\right\rangle\right\rangle\left\langle\tau_{\mathrm{L}}\right\rangle$. As shown in Fig. 3(a), the sensitivity $d<y>/ d s$ for the small $s$ increased as the noise was decreased. The evaluated sensitivity around $s=0 \mathrm{~V}$ was well explained by the theory from Eq. (2). The sensitivity also increased as the input threshold was increased, as shown in Fig. 3(b). The inputthreshold dependence of the sensitivity for the signal $s$ of $0.05 \mathrm{~V}$ is shown in Fig. 3(c). Even when the noise was the same, the sensitivity increased as the input threshold was increased. The good agreement between the theoretical and experimental behaviors confirmed the validity of the present model.

When the dynamics of the signal cannot be ignored, the output and the sensitivity given by Eqs. (1) and (2) are modified by the signal dynamics. Figure 4 shows the measured correlation coefficient $C_{1}$ between the target signal and output waveforms, where the target signal was a sinusoidal wave with a frequency of $2 \mathrm{kHz}$ and an amplitude of $0.025 \mathrm{~V}$. The Gaussian noise was imposed to the signal. The correlation showed a peak as a function of noise intensity, which was the typical behavior of stochastic resonance. When the input threshold increased, the peak position moved to the high side of the noise intensity. An important aspect was that the peak height almost maintained even when the peak position changed, which could not be explained by the previous theory. ${ }^{18)}$ 
In the context of our model, the input-output correlation coefficient is given by

$$
C_{1}=\frac{\rho(\theta) s}{2 \int_{\theta}^{\infty} \rho(\xi) d \xi}
$$

It is found that the correlation coefficient is also determined by the relative difference in transition probability induced by the input signal. Considering that the duty ratio of the output pulse determins the output power, the output SNR is found to have a similar form, $S N R=\rho(\theta) s / \int_{\theta}^{\infty} \rho(\xi) d \xi$. Our model suggests that the correlation coefficient also increases with increasing input threshold and it could well explain the observed threshold dependence of the correlation on the high side of the noise. Note that there is no arbitrary parameter in our model. On the other hand, the model does not suggest the decay of the curve in the left-hand side of the peak. Thereby, the dynamics of the input signal causes this discrepancy.

Then, we include the effect of the dynamics of the input signal into our model. To follow the input signal with frequency $f_{\mathrm{S}}$, the bistable system needs to satisfy the condition that the average period of the noise-induced output pulses is shorter than the input signal period; ${ }^{7,18)}\left\langle\tau_{\mathrm{H}}\right\rangle+\left\langle\tau_{\mathrm{L}}\right\rangle \leq 1 / f_{\mathrm{S}}$. When $\left.\left\langle\tau_{\mathrm{H}}\right\rangle+\left\langle\tau_{\mathrm{L}}\right\rangle\right\rangle 1 / f_{\mathrm{S}}$, the system cannot follow the input signal, thereby the correlation between the signal and the output is lost. Thus, the theoretical curve is truncated where $\left.\left\langle\tau_{\mathrm{H}}\right\rangle+\left\langle\tau_{\mathrm{L}}\right\rangle\right\rangle 1 / f_{\mathrm{S}}$ and the peak position is given by the noise intensity at which $\left\langle\tau_{\mathrm{H}}\right\rangle+\left\langle\tau_{\mathrm{L}}\right\rangle=1 / f_{\mathrm{S}}$. In consideration of Eq. (1), this condition implies that the peak position depends on the input threshold, noise bandwidth, and signal frequency.

The measured correlation coefficients for various combinations of signal frequency and noise bandwidth are shown in Figs. 5(a) and 5(b), respectively. The observed behaviors confirmed that the peak moved depending on both $f_{\mathrm{S}}$ and $B W_{\mathrm{N}}$, and a decrease in $f_{\mathrm{S}}$ and an increase in $B W_{\mathrm{N}}$ similarly contributed to the peak shift. Figure 5(c) shows the measured peak positions as a function of $f_{\mathrm{S}} / B W_{\mathrm{N}}$. A theoretical curve satisfying $\left\langle\tau_{\mathrm{H}}\right\rangle$ $+\left\langle\tau_{\mathrm{L}}\right\rangle=1 / f_{\mathrm{S}}$ is also shown. The theory well explains the experimental peak positions obtained from Figs. 5(a) and 5(b), confirming the validity of the model for the stochastic resonance peak position. The peak shift with almost-constant peak height shown in Fig. 4 was realized by the trade-off between the shift of the monotonically decreasing 
correlation-coefficient curve and the shift of the truncation position, which depended on the input threshold.

In conclusion, when the transition was frictionless, the sensitivity of the bistable system to a Gaussian-noise-imposed weak signal was significantly high at the high threshold because of the nonlinearity of the Gaussian distribution function. The origin of the high sensitivity is the divergence of the relative difference in Gaussian distribution function when the input threshold goes to infinity. The result shown here suggests that the nano- and molecular-scale bistable systems involve the mechanism efficiently detecting and handling a weak signal buried in noise.

\section{Acknowledgements}

S.K. would like to thank Prof. Hidetoshi Fukuyama of Tokyo University of Science, Japan, for his valuable comment and encouragement. This work was partly supported by MEXT KAKENHI Grant Number JP25110001 and JP25110013, JSPS KAKENHI Grant Number JP16K14240, and STARC FS program, Semiconductor Technology Academic Research Center (STARC), Japan. 


\section{References}

1) R. Benzi, Nonlin. Processes Geophys. 17, 431 (2010).

2) F. Moss, L. M. Ward, and W. G. Sannita, Clinical Neurophysiology 115, 267 (2004).

3) P. Hännggi, CHEMPHYSCHEM 3, 285 (2002).

4) I. Y. Lee, X. Liu, B. Kosko, and C. Zhou, Nano Lett. 3, 1683 (2003).

5) R. L. Badzey and P. Mohanty, Nature 437, 995 (2005).

6) S. Kasai and T. Asai, Appl. Phys. Express 1, 083001 (2008).

7) Y. Imai, M. Sato, T. Tanaka, S. Kasai, Y. Hagiwara, H. Ishizaki, S. Kuwabara, and T. Arakawa, Jpn. J. Appl. Phys. 53, 06JE01 (2014).

8) F. Hartmann, D. Hartmann, P. Kowalzik, A. Forchel, L. Gammaitoni, and L. Worschech, Appl. Phys. Lett. 96, 172110 (2010).

9) K. Nishiguchi and A. Fujiwara, Jpn. J. Appl. Phys. 50, 06 GF04 (2011).

10) K. Hayashi, S. de Lorenzo, M. Manosas, J. M. Huguet, and F. Ritort, Phys. Rev. X 2 , 031012 (2012).

11) Y. Hirano, Y. Segawa, T. Kuroda-Sowa, T. Kawai, and T. Matsumoto, Appl. Phys. Lett. 104, 233104 (2014).

12) K. Yoshida and K. Hirakawa, Nanotechnology 28, 125205 (2017).

13) R. F. Fox, Phys. Rev. A 39, 4148 (1989).

14) B. McNamara and K. Wiesenfeld, Phys. Rev. A 39, 4854 (1989).

15) H. Gang, G. Nicolis, and C. Nicolis, Phys. Rev. A 42, 2030 (1990).

16) M. I. Dykman, D. G. Luchinsky, R.Mannella, P. V. E. McClintock, N. D. Stein, and N. G. Stocks, Il Nuovo Cimento 17D, 661 (1995).

17) L. Gammaitoni, P. Hanggi, P. Jung, and F. Marchesoni, Rev. Mod. Phys. 70, 223 (1998).

18) A. R. Bulsara and L. Gammaitoni, Phys. Today 49, 39 (1996).

19) H. A. Kramers, Physica 7, 284 (1940).

20) P. Hänggi, P. Talkner, and M. Borkovec, Rev. Mod. Phys. 62, 251 (1990).

21) H. S. Chung and W. A. Eaton, Nature 502, 685 (2013).

22) K. Wiesenfeld and F. Jaramillo, Chaos 8, 539 (1998).

23) L. Gammaitoni, Phys. Rev. E 52, 4691 (1995).

24) M. M. Alibegov, Phys. Rev. E 59, 7079 (1999). 
25) A. Ichiki and Y. Tadokoro, Phys. Rev. E 87, 012124 (2013).

26) S. Kasai, Y. Tadokoro, and A. Ichiki, Phys. Rev. E 88, 062127 (2013).

27) D. Curtin, S. P. Hegarty, D. Goulding, J. Houlihan, Th. Busch, C. Masoller, and G. Huyet, Phys. Rev. E 79, 031103 (2004).

28) S. Fauve and F. Heslot, Phys. Lett. 97A, 5 (1983).

29) V. I. Melnikov, Phys. Rev. E 48, 2481 (1993).

30) F. Marchesoni, F. Apostolico, L. Gammaitoni, and S. Santucci, Phys. Rev. E 58, 7079 (1998). 


\section{Figure captions}

Fig. 1 (a, b) Potential landscapes and state transitions without and with friction, respectively. (c) Schematic input and output waveforms in a bistable system without friction and (d) resident time ratio as a function of input threshold from theory and numerical simulation for various noise distribution functions.

Fig. 2 (a) Input-output characteristics of an electronic bistable system effectively achieving frictionless transition by $\tau_{\mathrm{D}}<\tau_{\mathrm{AC}}<<1 / f_{\mathrm{S}}$ and examples of output waveform for Gaussian-noise-added DC signal. (b) Measured resident times vs. Gaussian noise intensity. Resident time ratio vs. (c) noise intensity and (d) input threshold. Theoretical curves are obtained by exactly computing Eq. (1).

Fig. 3 (a, b) Measured $\langle y\rangle$ as a function of input signal $s$ for various noise intensity and input thresholds, respectively. The solid lines show the exact theoretical curve obtained using Eq. (1) and the dotted lines show the curve approximated using Eq. (2). (c) Evaluated sensitivity as a function of input threshold.

Fig. 4 Measured input-output correlation coefficient for sinusoidal input as a function of noise intensity in the bistable system without friction. The amplitude and frequency of the signal are $0.025 \mathrm{~V}$ and $2 \mathrm{kHz}$, respectively. The dotted lines show the theoretical curve given by Eq. (4). The response in a linear system is also shown for comparison.

Fig. 5 ( $a, b)$ Stochastic resonance response for various input signal frequency $f_{S}$ and noise bandwidth $B W_{\mathrm{N}}$, respectively. The solid lines are guides for the eye. (c) Evaluated correlation coefficient peak position from (a) and (b). The theoretical curve is obtained from $\left\langle\tau_{\mathrm{H}}\right\rangle+\left\langle\tau_{\mathrm{L}}\right\rangle=1 / f_{\mathrm{S}}$ 


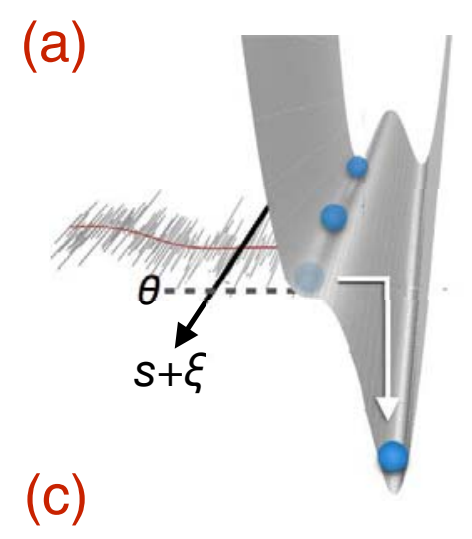

(c)
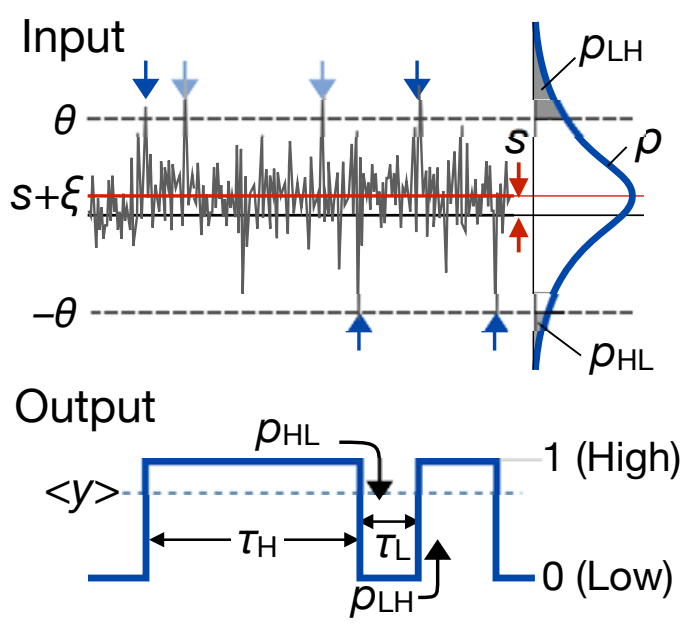

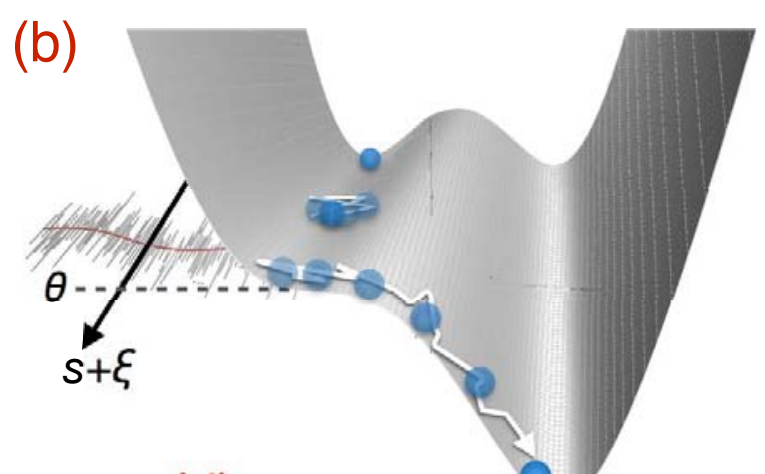

(d)

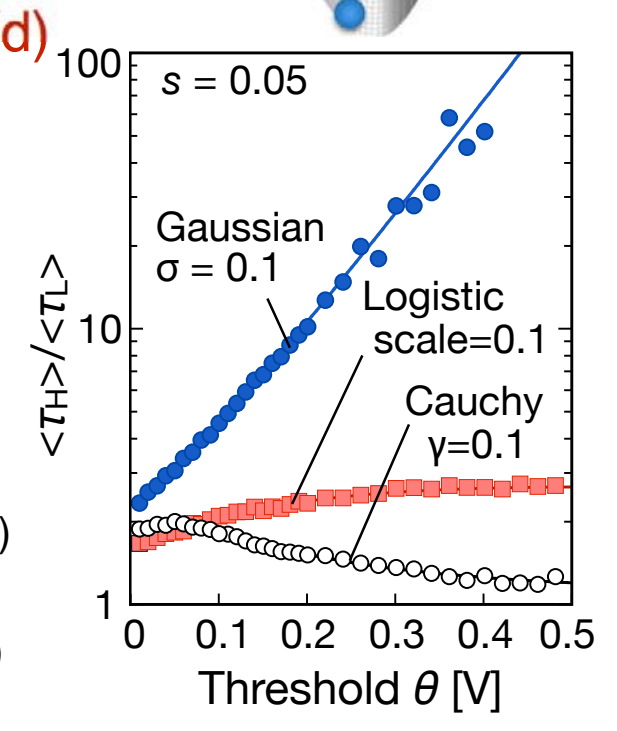

Figure 1 
(a)

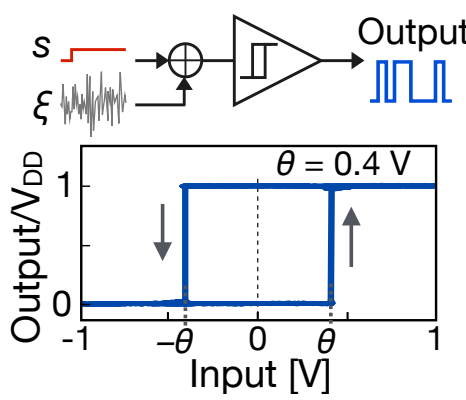

(b)
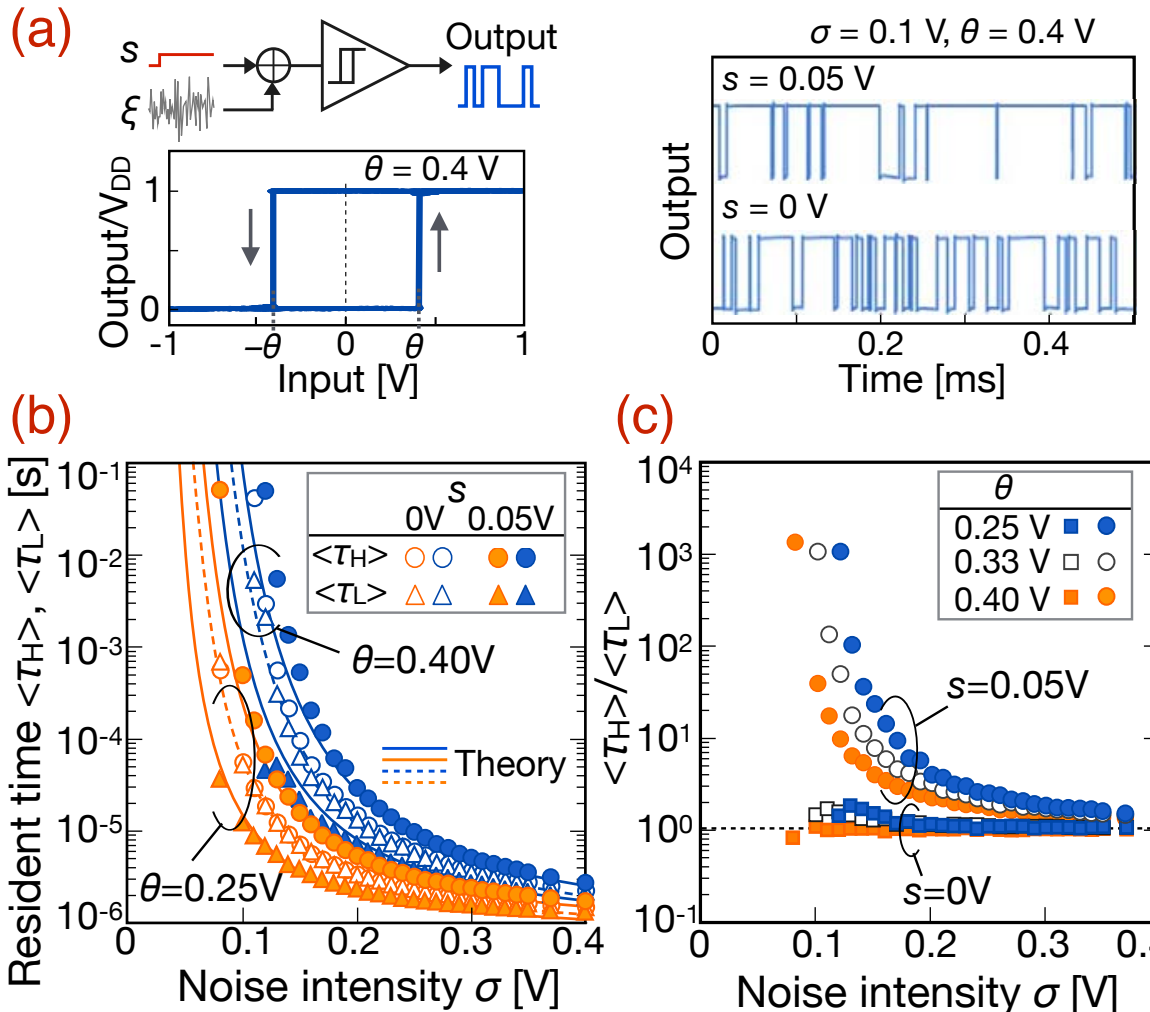

(c)
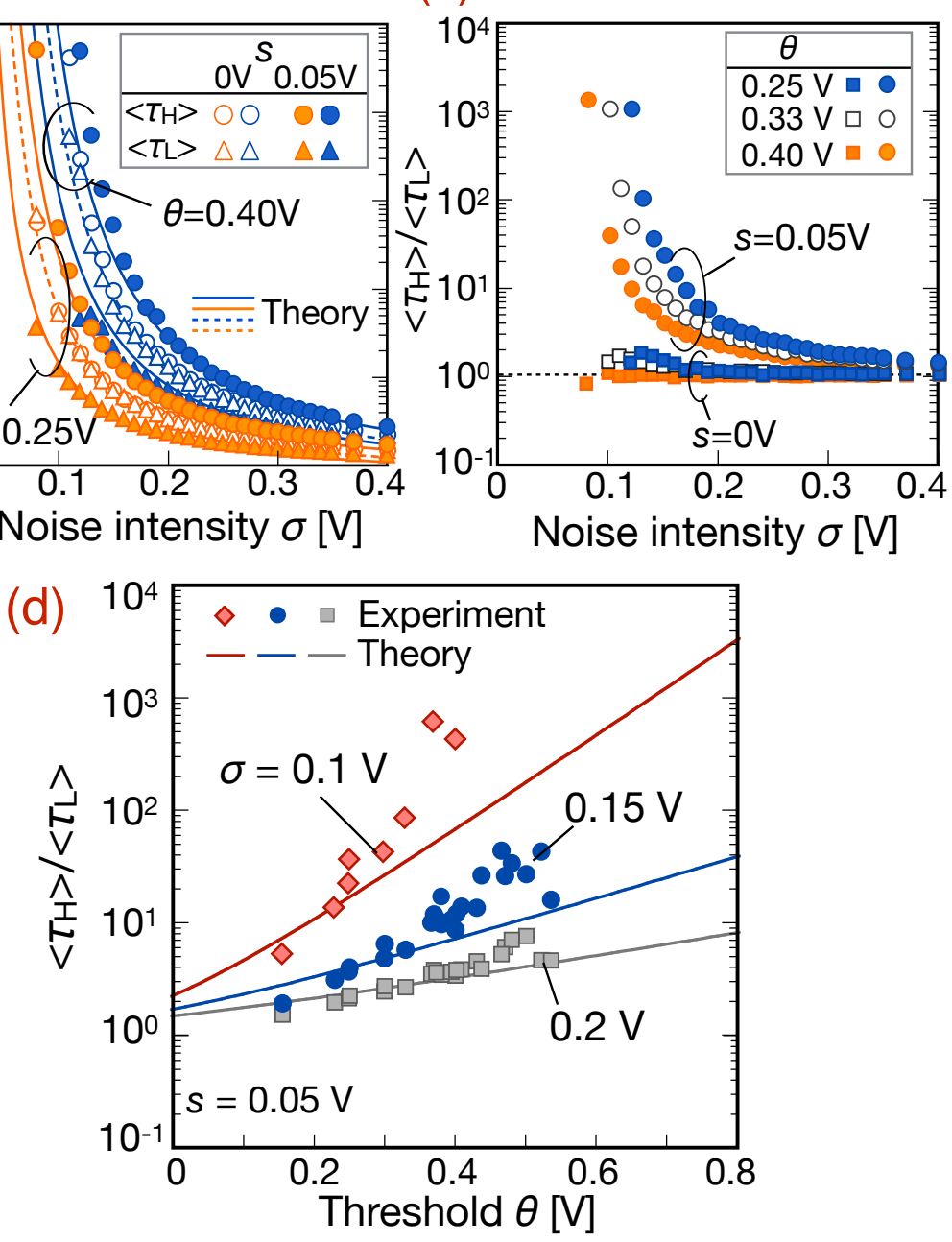

Figure 2 
(a)

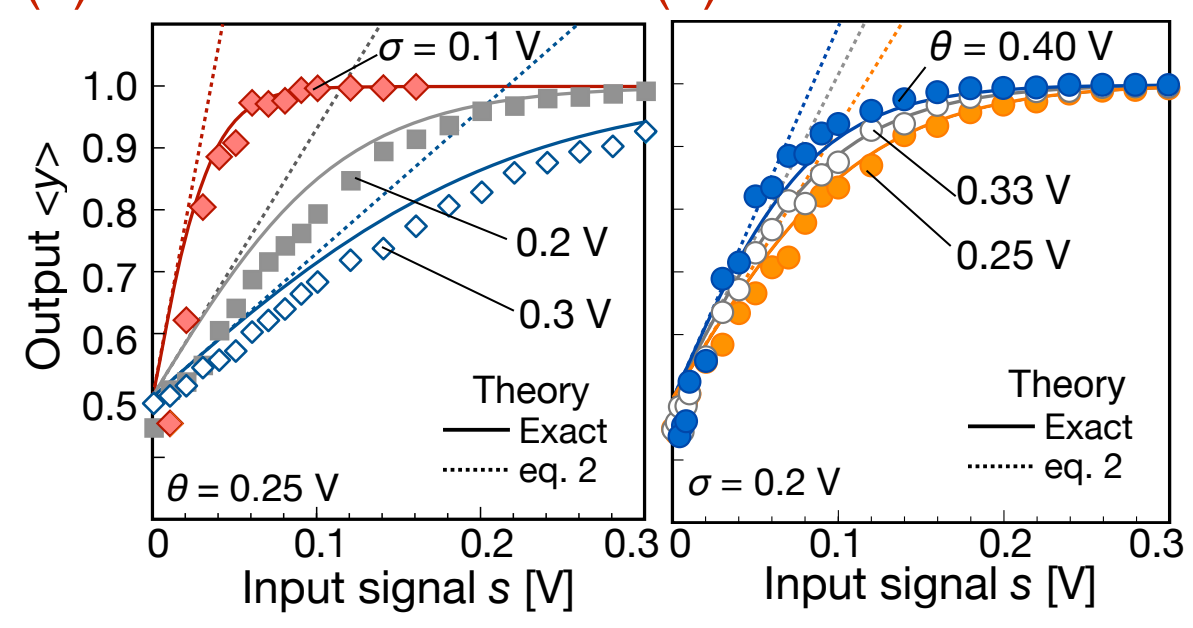

(c)

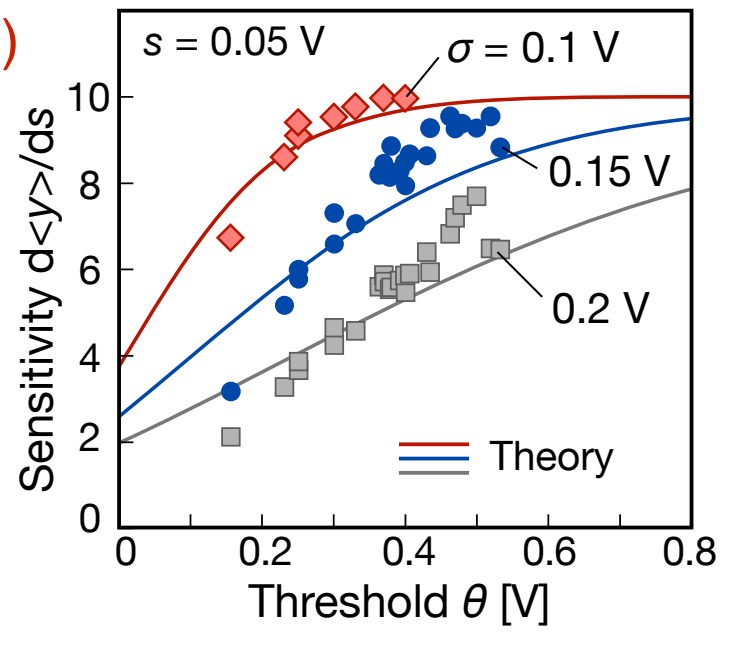

Figure 3 


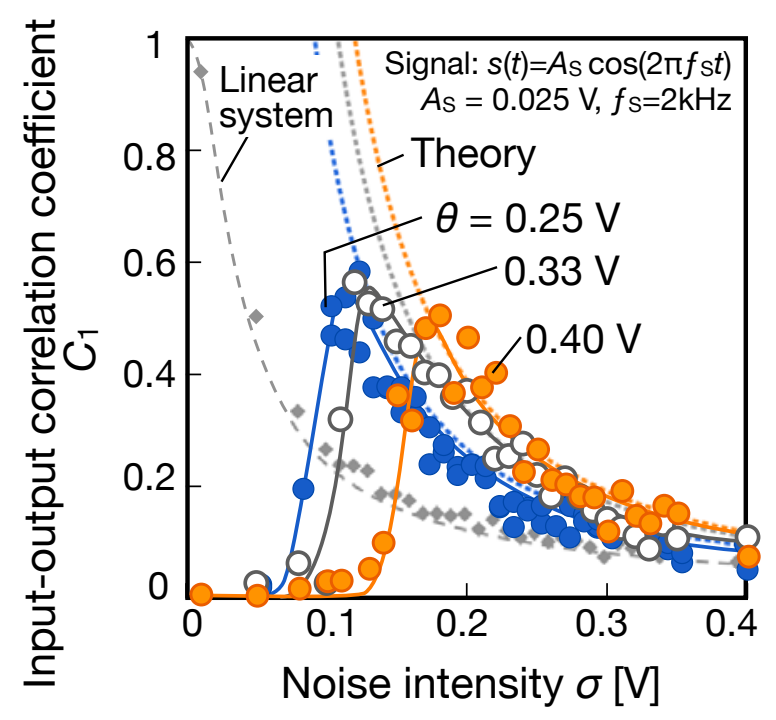

Figure 4 


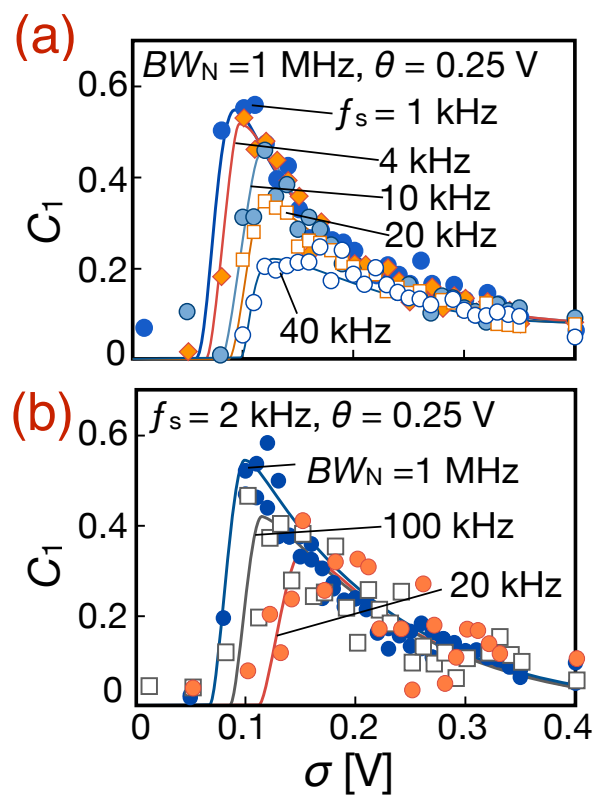

(c)

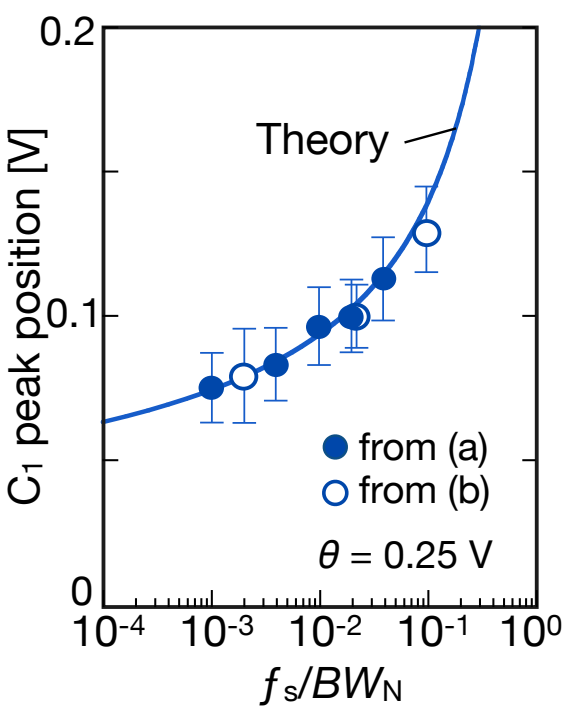

Figure 5 